Dear Author,

Please, note that changes made to the HTML content will be added to the article before publication, but are not reflected in this PDF.

Note also that this file should not be used for submitting corrections. 


\title{
Gait analysis in chronic heart failure: The calf as a locus of impaired walking capacity
}

\author{
Fausto A. Panizzolo a, Andrew J. Maiorana ${ }^{\mathrm{b}, \mathrm{c}}$, Louise H. Naylor ${ }^{\mathrm{a}}$, Lawrence Dembo ${ }^{\mathrm{b}, \mathrm{e}}$, \\ David G. Lloyd ${ }^{\mathrm{d}}$, Daniel J. Green ${ }^{\mathrm{a}, \mathrm{f}}$, Jonas Rubenson ${ }^{\mathrm{a}, *}$ \\ a The School of Sport Science, Exercise and Health, The University of Western Australia, Crawley, WA, 6009, Australia \\ ${ }^{\mathrm{b}}$ Advanced Heart Failure and Cardiac Transplant Service, Royal Perth Hospital, Perth, WA 6000, Australia \\ ' School of Physiotherapy and Exercise Science, Curtin University, Perth, WA 6102, Australia \\ ' Centre for Musculoskeletal Research, Griffith Health Institute, Griffith University, Gold Coast, QLD, 4222, Australia \\ envision Medical Imaging, Perth, Western Australia, Australia \\ ${ }^{\mathrm{f}}$ Research Institute for Sport and Exercise Science, Liverpool John Moores University, Liverpool, United Kingdom
}

\section{A R T I C L E I N F O}

Article history:

Accepted 14 September 2014

\section{Keywords:}

Muscle work

Gait mechanics

Heart failure

Triceps surae

\begin{abstract}
A B S T R A C T
Reduced walking capacity, a hallmark of chronic heart failure (CHF), is strongly correlated with hospitalization and morbidity. The aim of this work was to perform a detailed biomechanical gait analysis to better identify mechanisms underlying reduced walking capacity in CHF. Inverse dynamic analyses were conducted in CHF patients and age- and exercise level-matched control subjects on an instrumented treadmill at self-selected treadmill walking speeds and at speeds representing $+20 \%$ and $-20 \%$ of the subjects' preferred speed. Surprisingly, no difference in preferred speed was observed between groups, possibly explained by an optimization of the mechanical cost of transport in both groups (the mechanical cost to travel a given distance; $\mathrm{J} / \mathrm{kg} / \mathrm{m}$ ). The majority of limb kinematics and kinetics were also similar between groups, with the exception of greater ankle dorsiflexion angles during stance in CHF. Nevertheless, over two times greater ankle plantarflexion work during stance and per distance traveled is required for a given triceps surae muscle volume in CHF patients. This, together with a greater reliance on the ankle compared to the hip to power walking in CHF patients, especially at faster speeds, may contribute to the earlier onset of fatigue in CHF patients. This observation also helps explain the high correlation between triceps surae muscle volume and exercise capacity that has previously been reported in CHF. Considering the key role played by the plantarflexors in powering walking and their association with exercise capacity, our findings strongly suggest that exercise-based rehabilitation in CHF should not omit the ankle muscle group.
\end{abstract}

(c) 2014 Published by Elsevier Ltd.

\section{Introduction}

Chronic heart failure (CHF) is characterized by a marked reduction in walking capacity that in turn contributes to a reduction in quality of life (Juenger et al., 2002). Indeed, preferred walking speed has been reported to be $\sim 30 \%$ lower in CHF compared to healthy age-matched control groups (Beneke and Meyer, 1997; Figueiredo et al., 2013) and walking endurance decreases with increasing severity of the disease (Riley et al., 1992). Notably, walking capacity has also been directly linked with hospitalization and mortality rates in CHF (Forman et al., 2012).

\footnotetext{
* Corresponding author. Tel.: +61864885533.

E-mail address: jonas.rubenson@uwa.edu.au (J. Rubenson).
}

Identifying the mechanisms underlying the reduced walking performance in CHF therefore has important functional and clinical relevance. In many instances, skeletal muscle dysfunction, rather than cardiac function, is fundamental to the exercise intolerance evident in this group (Cohn et al., 1993; Cicoira et al., 2001; Fülster et al., 2013). This underlies the "skeletal muscle hypothesis" of exercise intolerance in CHF (Clark et al., 1996). However, most studies addressing the "skeletal muscle hypothesis" in humans have focused primarily on isolated skeletal muscle, including histology, biochemistry (Sullivan et al., 1990), morphology (Anker et al., 1997; Panizzolo et al., 2014) and strength (Lipkin et al., 1988; Toth et al., 2006). It is likely that these factors all contribute to some extent to reduced functional walking performance in CHF. Yet, unlike other conditions where skeletal muscle dysfunction purportedly leads to impaired walking, no study to date has examined the detailed biomechanics of walking itself in patients with CHF. Such an analysis would provide

http://dx.doi.org/10.1016/j.jbiomech.2014.09.015

0021-9290/( 2014 Published by Elsevier Ltd. 
an effective means to identify the end-effect of skeletal muscle dysfunction on walking mechanics in CHF. This information can help reveal the basis for functional limitations in CHF and foster evidencebased rehabilitation approaches aimed at restoring walking capacity.

The goal of this study was, therefore, to perform a detailed biomechanical gait analysis of walking in CHF patients, compared to healthy age-matched control participants. Recently, we identified that the plantarflexor muscles (the triceps surae) undergo proportionately more muscle wasting in CHF than other lower limb muscles (Panizzolo et al., 2014). Moreover, plantarflexor size, unlike the overall leg lean mass, is strongly correlated with peak aerobic capacity of walking in CHF patients (Panizzolo et al., 2014). These characteristics, together with the finding that the plantarflexors are the main source of work during gait in healthy young and old adults (McGowan et al., 2009; De Vita and Hortobagyi, 2000) and that a reduction in walking speed in older adults is related to the triceps surae function (Panizzolo et al., 2013), suggest that restrictions at the ankle joint might particularly affect the ability of CHF patients to achieve the typical gait speed and mechanics seen in a normal healthy population. Accordingly, we hypothesized that (1) a slower walking speed is selected in CHF, compared with healthy agematched individuals, to reduce total leg mechanical work; (2) the more pronounced wasting reported in plantarflexor muscles in $\mathrm{CHF}$ (Panizzolo et al., 2014) requires additional redistribution of mechanical work during stance from the ankle to the other lower limb joints; and (3) this redistribution would be more pronounced at faster walking, where a greater percentage of peak aerobic capacity is utilized. This hypothesis is based on the strong correlation between plantarflexor muscle size and strength and peak aerobic capacity during walking previously reported in CHF (Panizzolo et al., 2014), and the possibility therefore that mechanical work is "shunted" from the ankle to other less aerobically limiting muscles at other joints.

\section{Methods}

\subsection{Subjects}

We recruited 10 subjects ( 6 men, 4 women) with CHF (NYHA class II-IV; ejection fraction $=30.9 \pm 9.7 \%$, mean \pm S.D. $)$ and 11 healthy subjects from the local community $(8$ men, 3 women; see Table 1 for subjects characteristics). Exclusion criteria for the CHF population are presented in the Supplementary Material. All subjects were free from musculoskeletal injury and other musculoskeletal diseases and provided written informed consent prior to participating in the study. All procedures were approved by the Human Research Ethics Committee at The University of Western Australia and Royal Perth Hospital.

\subsection{Preferred walking speed}

A protocol based on over-ground and treadmill walking trials was used to define each subjects' preferred treadmill walking speed. The detailed description of this protocol is presented in Panizzolo et al. (2013) and in the Supplementary Material.

\subsection{Joint and lower limb mechanical work and cost of transport (COT)}

Biomechanical measurements were collected with subjects wearing their own sport shoes while walking on an instrumented split-belt treadmill measuring 3D ground reaction forces (Bertec, Columbus, $\mathrm{OH}, \mathrm{USA} ; 2000 \mathrm{~Hz}$ ) at three different

Table 1

Subject characteristics. Data are means \pm SD.

\begin{tabular}{llll}
\hline Group & Age [yr] & Height $[\mathrm{m}]$ & Weight $[\mathrm{kg}]$ \\
\hline Control & $63.1 \pm 5.6$ & $1.73 \pm 0.06$ & $70.1 \pm 8.8$ \\
CHF & $60.7 \pm 9.8$ & $1.67 \pm 0.10$ & $73.0 \pm 19.0$ \\
\hline
\end{tabular}

The chronic heart failure (CHF) group underwent regular exercise activity 2-3 times per week for $\sim 1$ hour per session (treadmill walking and resistance weight training) for a duration of $3.8 \pm 1.3$ months as part of their standard patient care. The control subjects underwent similar levels of weekly exercise. walking speeds: the subjects' preferred speed, a speed $20 \%$ faster than their preferred speed and a speed 20\% slower than their preferred speed. Subjects walked at each testing speed for approximately $30 \mathrm{~s}$ to $1 \mathrm{~min}$ before data collection.

Three-dimensional (3D) gait analysis was performed on each subject during their treadmill walking trials. The marker set and configuration used for 3D motion capture (VICON, Oxford Metrics, UK; $100 \mathrm{~Hz}$ ) was similar to that of Besier et al (2003), with the addition of torso markers (a full description of the marker set is provided in the Supplementary Material). Functional joint centers for the hip and knee were defined using the procedures of Besier et al. (2003). All markers and force trajectories were filtered using a zero-lag fourth-order low-pass Butterworth filter with a $5-7 \mathrm{~Hz}$ optimal cut-off frequency that was selected using a custom residual analysis algorithm (MATLAB, The MathWorks Inc., USA).

Marker positions collected during a static trial were used to generate subjectspecific musculoskeletal models in OpenSim 2.0.2 (Delp et al., 2007). The generic OpenSim musculoskeletal model (Arnold et al., 2010) was scaled using an inverse kinematics algorithm based on the position of the markers placed on anatomical landmarks and on the functional joint centers previously determined. Joint angles, net moments and instantaneous powers were computed using inverse kinematics and inverse dynamics performed in the joint coordinate systems of the scaled mode for the walking trials. These calculations were made directly in OpenSim by combining 3D markers trajectories and measured ground reaction forces. Positive joint work was computed for the stance phase and for the complete stride by integrating the positive values of the instantaneous joint power traces (further details of the joint mechanical measurements are provided in the Supplementary Material). The total positive work in the lower limbs was computed both for the stance phase and for the entire stride from the sum of each joint (left and right legs were computed individually and summed) and normalized to the lower limb lean mass (see below). The distribution of total work between individual joints was computed by dividing the total lower limb work by the positive work in the individual joints (sum of left and right joints). The total mechanical cost of transport (COT) $\mathrm{J} / \mathrm{kg} / \mathrm{m}$ was calculated by dividing the lower limb lean mass-normalized positive work over the stride by the distance traveled over the stride.

The work produced in plantarflexion during stance was computed separately and normalized to the triceps surae volume (see below). Similarly, the specific COT for ankle plantarflexion was computed as the sum of the left and right triceps surae volume-specific positive ankle plantarflexion joint work divided by the distance traveled over one stride. Five non-consecutive strides per speed were used for generating mean kinematic, kinetic and work data for each individual subject, which were subsequently combined to calculate group mean data.

2.4. Lean lower limb mass (DXA) and plantarflexor volume (3Dultrasound)

Overall body composition was determined using DXA (Luna Prodigy, EnCore 2004, GE Medical Systems, Madison, WI, USA) on each subject. Lean mass for the lower limbs was computed separately by selecting a region of interest from the great trochanter to the pubic symphysis and including the leg and foot.

Plantarflexor volume was computed using a three-dimensional ultrasound technique (3DUS) based on a combination of B-mode ultrasound imaging and 3D motion data, the procedures of which have been described in detail in Barber et al. (2009) and Panizzolo et al. (2014) and are reported in the Supplementary Material.

\subsection{Peak and sub-maximal oxygen consumption}

On a separate testing day, peak oxygen uptake $\left(\dot{V} \mathrm{O}_{2}\right.$ peak) was assessed using an incremental walking protocol on a motorized treadmill (Panizzolo et al., 2013). Indirect calorimetry was conducted using a Vmax Encore gas analysis system (Sensormedics, Yorba Linda, California), which enabled the measurement of expired gas concentrations and volumes. Absolute $\dot{V} \mathrm{O}_{2}$ peak was expressed in $\mathrm{ml} / \mathrm{min}$ and normalized to body mass $(\mathrm{ml} / \mathrm{kg} / \mathrm{min})$. Sub-maximal oxygen consumption at the three subject-specific speeds described above were also obtained.

\subsection{Statistical analysis}

A $3 \times 2$ mixed model repeated measures ANOVA was performed to evaluate differences between the control and CHF groups across the three testing speeds. The significance level was set at $p<0.05$ (SPSS Inc., Statistics 21, USA). ANOVA analyses included joint kinematics (minimum, maximum and total angle range) and kinetics (peak moments), the positive work in the combined lower limb (normalized by lower limb lean mass) and the plantarflexor work at the ankle normalized to the triceps surae volume. We analyzed both the mechanical work performed during the stride and stance phase, as well as the mechanical cost of transport (work per distance travelled). Main effects of group and speed and interaction effects were evaluated in these analyses. The distribution of work produced across joints was evaluated with a $3 \times 2$ MANOVA (significant level of $p<0.05$ ) including the three lower limb joints. An arcsin conversion was applied to the percentage joint contribution prior to performing this analysis. Joint and speed were set as multivariate factors and group as a univariate factor. Where significant main and/or interaction effects were detected in ANOVA or MANOVA analyses a Bonferroni post hoc test was conducted. 


\section{Results}

\subsection{Preferred walking speed and spatio-temporal parameters}

Preferred walking speed on the treadmill and over-ground were not significantly different between groups. Spatial-temporal gait parameters including stride length, stride frequency, duty factor, stance and swing times were similarly not significantly different between groups (Table 2).

\subsection{Joint kinematics and kinetics}

No differences were found between CHF and control participants in peak joint moments or powers at the ankle, knee or hip across all three speeds. A main effect of group was reported in the kinematics of the ankle. The CHF group exhibited a higher dorsiflexion peak in stance $(p=0.01)$ but no differences in the total ankle range of motion $(p=0.2)$. Joint kinematics, kinetics and power traces for the preferred speed are displayed in Fig. 1.

\subsection{Total lower limb lean mass-specific and plantarflexor volume-specific work and COT}

Total leg lean mass-specific mechanical work and COT across the stride were on average $10.2 \%$ and $15.6 \%$ greater in CHF versus control (Fig. 2), although no statistical main effect of group was observed in either of these parameters ( $p=0.2$ and $p=0.1$, respectively). A significant main effect of speed was present in both lower limb lean

Table 2

Spatio-temporal parameters at different testing speeds. Data are means \pm SD

\begin{tabular}{|c|c|c|c|c|c|c|c|}
\hline Speed & Group & Testing speed $[\mathrm{m} / \mathrm{s}]$ & Stance time [s] & Swing time [s] & Duty factor & Stride freq. [Hz] & Stride length [m] \\
\hline Slow & $\begin{array}{l}\text { Control } \\
\mathrm{CHF}\end{array}$ & $\begin{array}{l}0.81 \pm 0.13 \\
0.79 \pm 0.20\end{array}$ & $\begin{array}{l}0.77 \pm 0.07 \\
0.80 \pm 0.12\end{array}$ & $\begin{array}{l}0.51 \pm 0.06 \\
0.51 \pm 0.07\end{array}$ & $\begin{array}{l}0.60 \pm 0.01 \\
0.61 \pm 0.03\end{array}$ & $\begin{array}{l}0.79 \pm 0.08 \\
0.78 \pm 0.10\end{array}$ & $\begin{array}{c}1.03 \pm 0.14 \\
0.96 \pm 0.21\end{array}$ \\
\hline Preferred & $\begin{array}{l}\text { Control } \\
\text { CHF }\end{array}$ & $\begin{array}{l}1.00 \pm 0.16 \\
0.99 \pm 0.26\end{array}$ & $\begin{array}{l}0.69 \pm 0.06 \\
0.74 \pm 0.13\end{array}$ & $\begin{array}{l}0.48 \pm 0.05 \\
0.48 \pm 0.06\end{array}$ & $\begin{array}{l}0.59 \pm 0.01 \\
0.61 \pm 0.02\end{array}$ & $\begin{array}{l}0.87 \pm 0.08 \\
0.84 \pm 0.13\end{array}$ & $\begin{array}{l}1.16 \pm 0.16 \\
1.11 \pm 0.22\end{array}$ \\
\hline Fast & $\begin{array}{l}\text { Control } \\
\mathrm{CHF}\end{array}$ & $\begin{array}{l}1.21 \pm 0.21 \\
1.19 \pm 0.31\end{array}$ & $\begin{array}{l}0.64 \pm 0.05 \\
0.67 \pm 0.12\end{array}$ & $\begin{array}{l}0.45 \pm 0.04 \\
0.46 \pm 0.07\end{array}$ & $\begin{array}{l}0.59 \pm 0.01 \\
0.59 \pm 0.02\end{array}$ & $\begin{array}{l}0.93 \pm 0.07 \\
0.91 \pm 0.14\end{array}$ & $\begin{array}{l}1.28 \pm 0.18 \\
1.23 \pm 0.24\end{array}$ \\
\hline
\end{tabular}

a
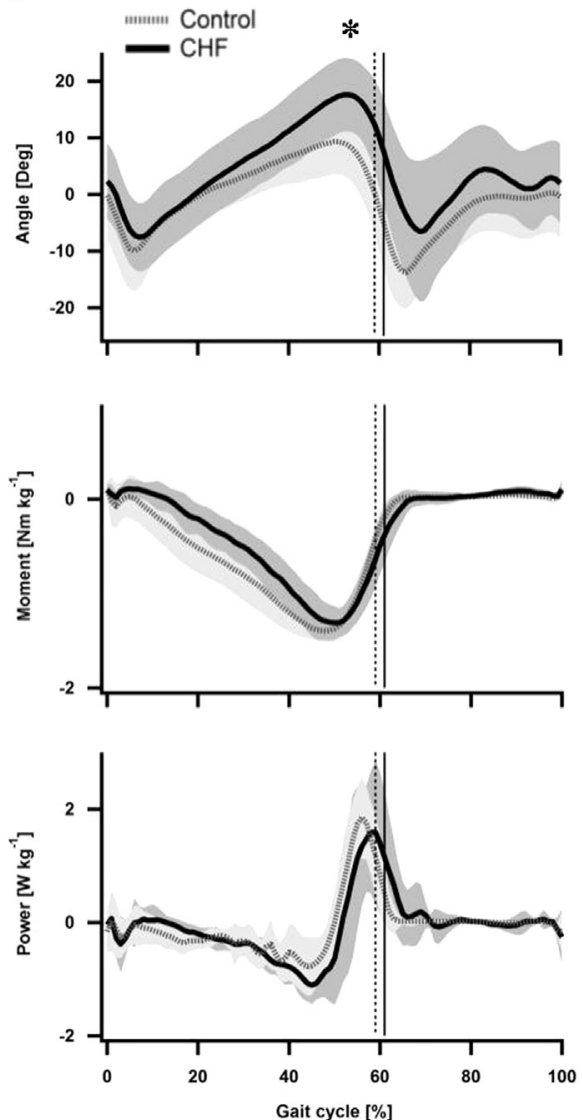

b
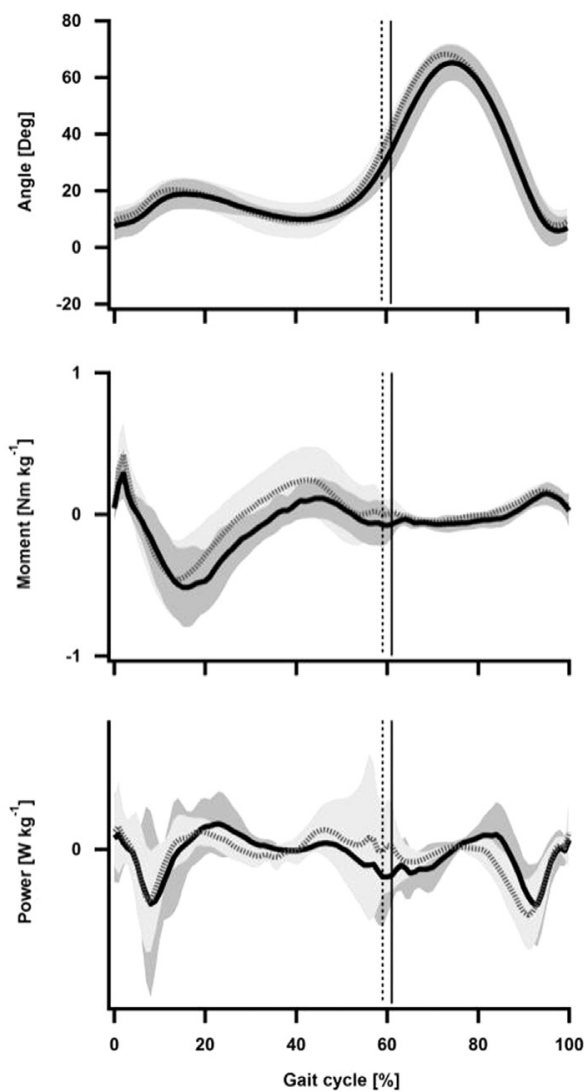

C
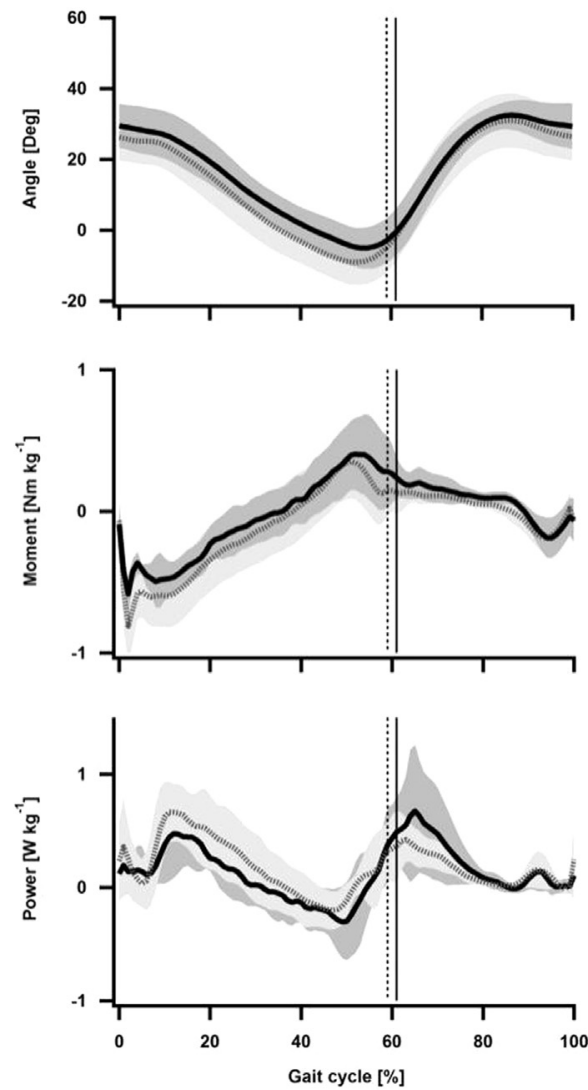

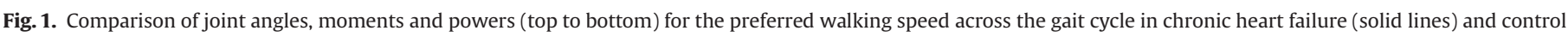

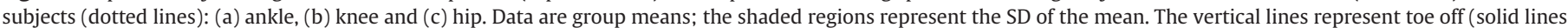

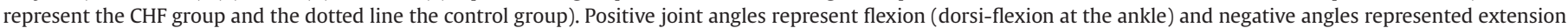

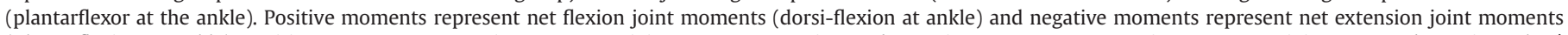

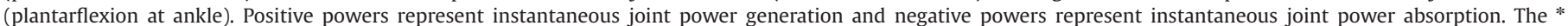
represents statistically different ankle joint dorsiflexion in stance $(p<0.05)$. 
a

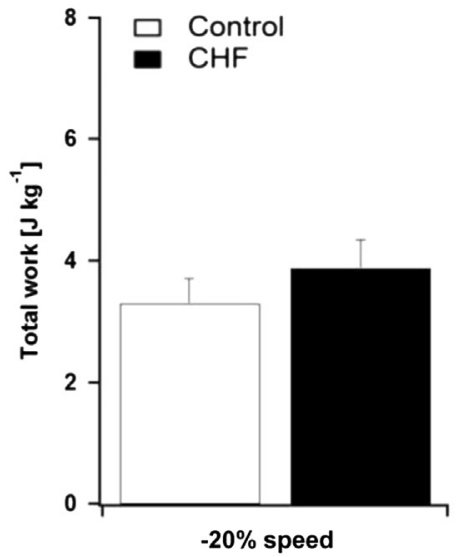

b

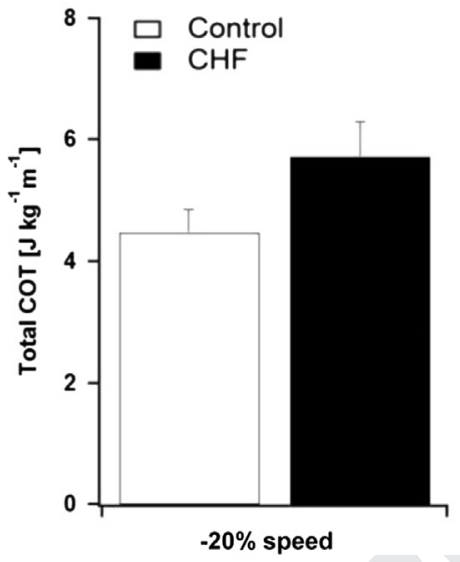

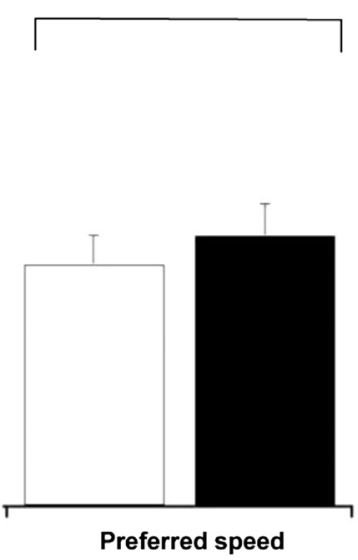

Preferred speed

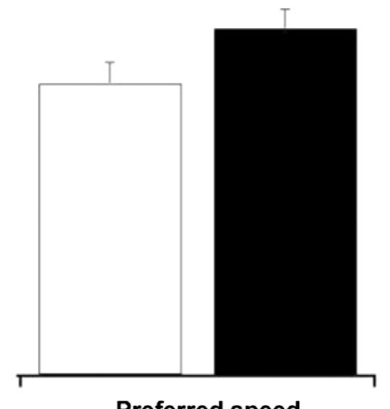

Preferred speed

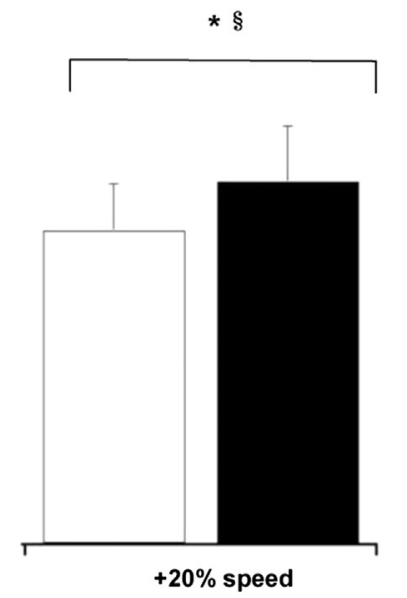

$\star \S$

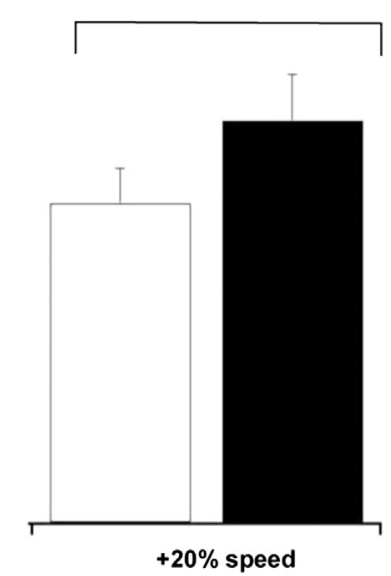

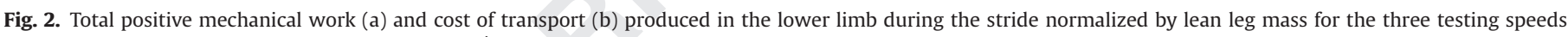

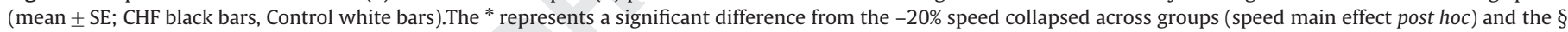
represents a significant difference from the preferred speed collapsed across groups (speed main effect post hoc).

mass-specific total work $(p<0.001)$ and COT $(p<0.001)$. Post hoc tests revealed that when collapsed across groups differences were present between all speeds for lower limb lean mass-specific total work. Post hoc tests also revealed that the slowest and preferred speeds did not differ in lower limb lean mass-specific COT, but that each was lower than the COT at the fastest speed. No significant interaction effects for positive leg lean mass-specific work or COT were present. The same statistical findings existed for the total leg work when normalized to body mass.

A main effect of group on the triceps surae volume-specific plantarflexion work and COT in stance was observed (Fig. 3); $p=0.024$ and $p=0.008$, respectively. The amount of work produced by the CHF group was $52.8 \%, 69.2 \%$ and $69.2 \%$ higher than the control group (for the three testing speeds); the COT was also $68.4 \%, 74.0 \%$ and $54.4 \%$ higher than the control group (for the three testing speeds). A main effect of speed was observed on the triceps surae volume-specific plantarflexion work and COT in stance ( $p<0.001$ and $p=0.002$, respectively). Post hoc tests revealed that when collapsed across groups the triceps surae volume-specific plantarflexion work were different from each other between all speeds and that the COT at the slowest and preferred speeds were not different from each other but both were lower than that at the fastest speed. No interaction effect was observed between group and speed ( $p=0.2$ and $p=0.3$, respectively).

\subsection{Distribution of joint work}

Across all the testing speeds the hip produced the highest amount of positive work, while the knee joint produced the least work, in both control and CHF groups. Nevertheless, a different distribution of work during stance was found between the CHF and the control group (Fig. 4). Main multivariate effects of group and speed on work distribution were not present $(p=0.3$ and $p=0.08$, respectively) but an interaction effect was found between joint and group $(p=0.01)$ and between speed and joint $(p=0.005)$. Post hoc analyses reveled differences in the percent work between groups at the ankle at all speeds ( $p=0.011, p=0.023$, and $p=0.001$ ) and at the hip only at the slowest speed $(p=0.044, p=0.13$ and $p=0.055)$. These results reflected that the percentage of work produced at the ankle joint was higher in the CHF, while an opposite trend was found for the hip, and that the ankle produced proportionately more work in CHF vs. control participants as speed increased (Fig. 4).

\subsection{Aerobic capacity}

$\dot{V} \mathrm{O}_{2}$ peak was significantly higher $(p<0.001)$ in the control group than in CHF $(35.8 \pm 7.9$ and $15.8 \pm 2.8 \mathrm{ml} / \mathrm{kg} / \mathrm{min}$, respectively). While no differences were reported in the sub-maximal oxygen consumption values between groups at any of the testing 


\section{a}

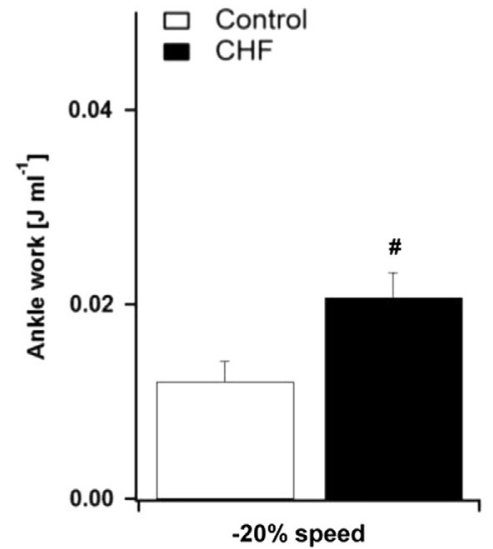

b

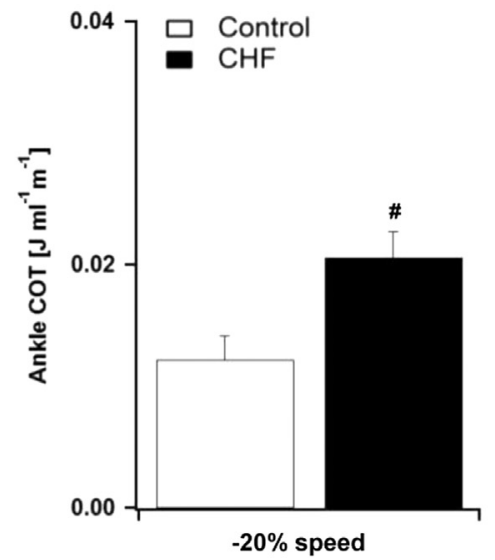

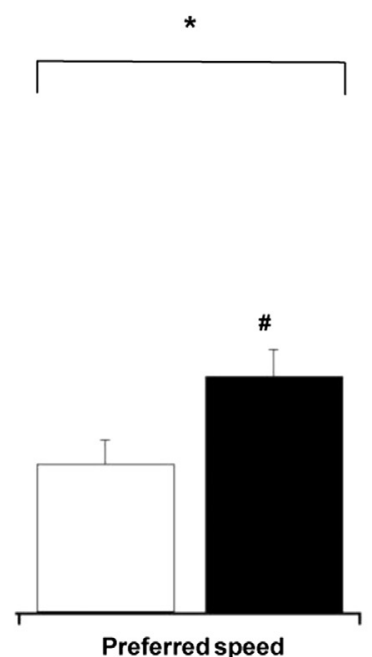

Preferred speed

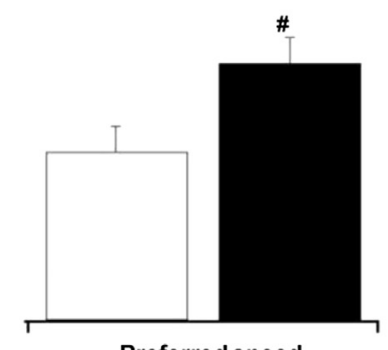

Preferred speed

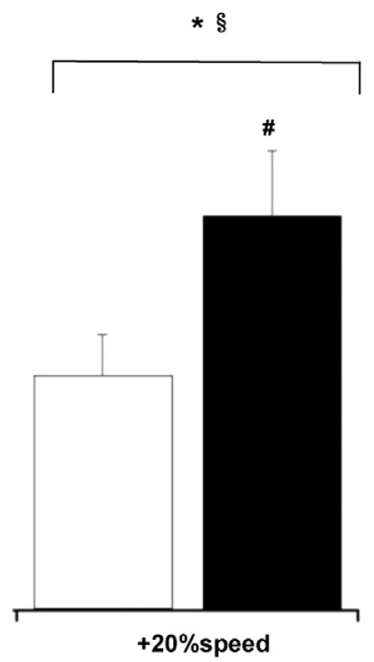

$+20 \%$ speed

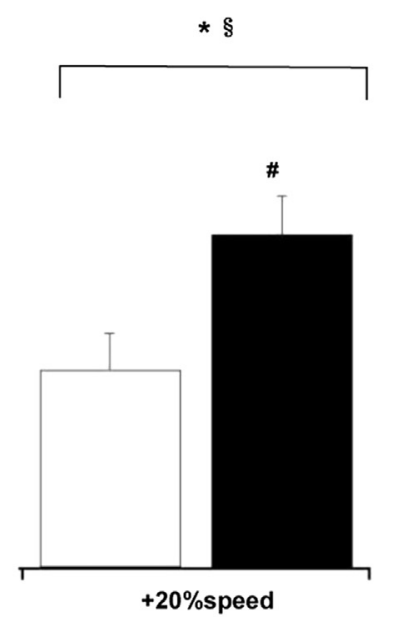

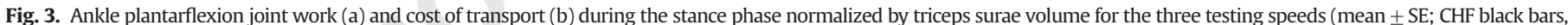

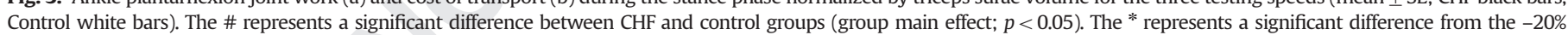

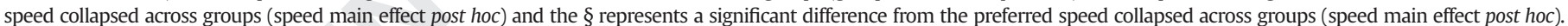

speeds, a main effect of group $(p<0.001)$ and a main effect of speed $(p=0.002)$ were reported when oxygen consumption was expressed as a percentage of maximum aerobic capacity (Table 3 ).

\section{Discussion}

\subsection{Speed and overall gait mechanics}

One reason why CHF patients may have previously exhibited reduced walking function, including speed, is to compensate for lower limb muscle mechanical capacities (Clark et al., 1996; Harrington et al., 1997; Toth et al., 2006). Our first hypothesis that a reduction in preferred speed is present and is associated with equal levels of mechanical work compared to a faster walking healthy control group was, however, not met. Surprisingly, the preferred speed, both over-ground and on the treadmill, were not statistically different between groups, nor were the spatio-temporal gait parameters. Interestingly, the similarity in gait function between the CHF and control groups extended to joint kinetics and kinematics, with few clearly discernable differences in either joint angles, net joint torques or power with the exception of a more dorsiflexed ankle joint in CHF patients (Fig. 1). One possible explanation for the lack of difference in preferred speed in our study compared to others (Beneke and Meyer, 1997; Figueiredo et al., 2013) might be the training status of our patients. Because our CHF patients underwent moderate levels of treadmill exercise it is possible that their similar walking speed was due to a training effect (Beneke and Meyer, 1997). Thus, despite the CHF patients presenting with severely limited $\dot{V} O_{2}$ peak, regular training may possibly promote similar submaximal walking patterns. It remains possible, therefore, that more pronounced differences in preferred speed and joint mechanics would be detected if CHF patients without training were analyzed. Another, associated reason for our lack of difference in walking speed, versus previous studies, is the disease severity. If previous studies included subjects with more severe CHF (e.g. NYHA III-IV), then they may have been less capable of achieving "normal," albeit compensated, walking speeds.

\subsection{Relative muscular work and distribution of joint work}

The similarity in speed and joint mechanics at first hand suggest that there are no major biomechanical gait differences present in CHF patients. However, a second possible strategy to offset the 
a

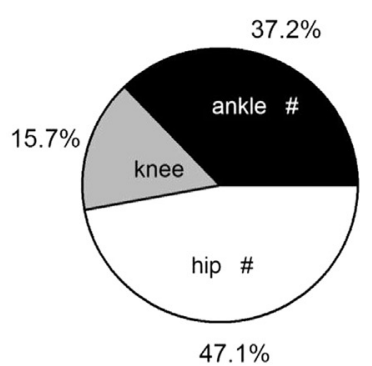

b

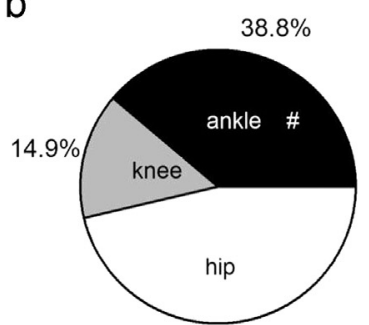

$46.3 \%$

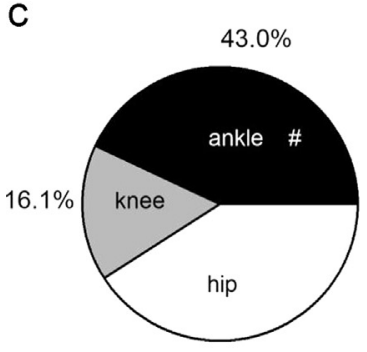

$40.9 \%$
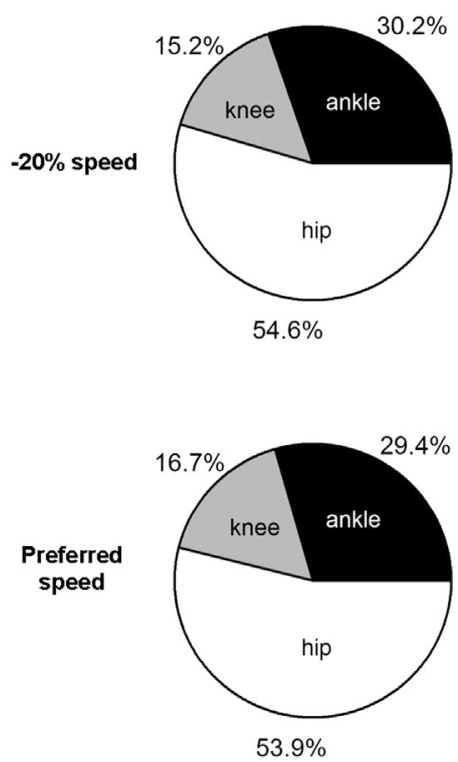

Control

Fig. 4. The distribution of positive mechanical work (group mean data) across lower limb joints at the three different testing speeds. (a) slower than preferred, (b) preferred and (c) faster than preferred speeds. The \# represents significant differences (MANOVA post hoc analyses) between groups at the specific joints.

Table 3

Rate of oxygen consumption expressed as a percentage of the peak oxygen consumption $\left(\dot{V}_{2}\right.$ peak) for the three testing speeds. Data are means $\pm \mathrm{SD}$.

\begin{tabular}{lll}
\hline Speed & Group & Relative $\dot{V}_{2}$ peak [\%] \\
\hline \multirow{2}{*}{ Slow } & Control & $28.0 \pm 0.1$ \\
& CHF & $55.0 \pm 0.1$ \\
Preferred & Control & $30.1 \pm 0.1$ \\
& CHF & $64.3 \pm 0.1$ \\
Fast & Control & $34.0 \pm 0.1$ \\
& CHF & $65.7 \pm 0.1$ \\
\hline
\end{tabular}

pronounced loss of muscle mass in the triceps surae of the CHF patients (Panizzolo et al., 2014) is to redistribute work from the ankle to other joints (DeVita and Hortobagyi, 2000). Contrary to this hypothesis, we found that there was an increase in the proportion of work performed at the ankle in CHF. Because of the reduced size of the plantarflexor muscles in CHF, this results in muscle volumespecific work that is over twice that in the healthy control group (Fig. 3a). Therefore, CHF patients apparently choose not to compensate for their reduced plantarflexor muscle volume, but instead to work at substantial higher relative effort (for their available muscle mass) at the ankle. Considering that the ankle is a key joint in producing the mechanical work of walking (McGowan et al., 2009), the much higher joint work per unit of muscle volume may influence both the acute effort during the stance phase $(\mathrm{J} / \mathrm{ml}$ over the stance, Fig. 3a) as well as the effort to travel a given distance $(\mathrm{J} / \mathrm{ml} / \mathrm{m}$ Fig. 3b). These factors may be a primary cause of the earlier symptoms of walking fatigue characteristic of this population (Clark et al., 1996), and may be one important limitation to walking aerobic capacity. It is intriguing to consider whether the higher dorsiflexion angles during stance in CHF (Fig. 1) also lead to altered in vivo muscle mechanics. Rubenson et al. (2012) reported operating lengths of the soleus in healthy young adults that are conserved to the ascending limb of the force-length curve during gait. The higher dorsiflexion angles in CHF may lead to longer muscle lengths that generate passive forces, thus partly compensating for the reduced active force capacity of the muscle (although possibly at the increased risk of muscle strain injury). At present this mechanisms remains speculative and requires further in vivo analyses to test.

Surprisingly, the greater demand on the ankle to produce the limb mechanical work increased, rather than decreased, with speed (Figs. 3a and 4), further taxing the small triceps surae muscle mass. Our earlier work on the triceps surae in CHF established a strong link between muscle size and $\dot{\mathrm{V}} \mathrm{O}_{2}$ peak (Panizzolo et al., 2014), leading to our prediction that mechanical work would be "shunted" away from the ankle as CHF patients walk closer to their aerobic capacity, thus potentially shifting reliance on to other less aerobically limiting muscle groups. The finding that the opposite occurs may in fact help to explain the strong link between the size of the triceps surae and $\dot{V O}_{2}$ peak during walking. Indeed, these muscles were key in powering walking, compared to the control group that relied more on the hip, and were responsible for the majority of mechanical work during fast walking.

\subsection{What dictates preferred walking speed and joint work modulation in $\mathrm{CHF}$ ?}

Our results offer some intriguing questions about what factors influence the self-selected walking mechanics in CHF. Firstly, why did the CHF patients not walk slower despite their reduction in muscle size and impaired aerobic capacity? A possible explanation may be an optimization of speed relative to the mechanical COT. We found that the mechanical work required to travel a given distance was not reduced when shifting from the preferred speed to a slower walking speed in either group (Fig. 2b). On the other hand, the COT was elevated in both groups when walking faster than the preferred speed. In this regard, the self-selected speed may represent the fastest speed possible before increasing the mechanical COT. This is plausible since the cumulative (repetitive) loading affects fatigue and energy use in the musculature. Mechanical gait optimization may represent an alternative, or possibly parallel, determinant of gait speed to the respiratory optimization recently reported in CHF patients (Figueiredo et al., 2013).

Secondly, why do CHF patients rely on the ankle to a greater extent to power walking compared to healthy age-matched adults? It has been established previously that aging causes an increased reliance on the hip to power walking (De Vita and Hortobagyi, 2000; Silder et al., 2008). Our data on healthy older adults supports this, with over $50 \%$ of the work attributed to the hip in this group (Fig. 4). It is not entirely clear why, in the CHF group, the contributions of each joint to the total mechanical work resemble those in healthy young adults (Farris and Sawicki, 2012), especially considering the reduced muscle volume of the ankle plantarflexors. A first possible explanation might be found in the hip flexor function. These muscle groups have been found to be weaker in CHF (Toth et al., 2006; Toth et al., 2010; Minotti et al., 1993), or functionally impaired. On the other hand, previous studies (Harridge et al., 1996; Carrington et al., 2001; Panizzolo et al., 2014) indicate that the strength of ankle plantarflexors, despite their prominent loss of muscle volume, may 
be less affected in CHF compared to hip muscle strength. Thus, a greater reliance on the ankle may reflect a more pronounced loss of the muscle force capacity at the hip in CHF compared to healthy older adults.

\subsection{Summary}

The preferred speed and overall joint kinematics and kinetics are similar between CHF and age- and exercise level-matched subjects. Nevertheless, a marked increase in the muscle volumespecific plantarflexion work and a greater reliance on the ankle over other joints to power walking in CHF patients may help explain their decreased walking capacity. The present study strengthens the finding that the plantarflexor muscles (triceps surae) are a key muscle group limiting exercise capacity (Panizzolo et al., 2014) and should be included in the design of exercise-based rehabilitation specific to this population.

\section{Conflict of interest}

The authors declare that no conflict of interest exists with any of the authors of this article.

\section{Uncited reference}

Treece et al., (2003).

\section{Acknowledgments}

This work was supported by a Grant-in-Aid (G09P 4469) from the National Heart Foundation of Australia to J.R., D.J.G., A.J.M. and D.G.L., and a thesis dissertation grant from the International Society of Biomechanics to F.A.P. The authors would like to acknowledge Ms. Kirsty McDonald and Mr. Timothy Henry for their help during data collection, and all of the participants who volunteered for this study.

\section{Appendix A. Supporting information}

Supplementary data associated with this article can be found in the online version at http://dx.doi.org/10.1016/j.jbiomech.2014.09.015.

\section{References}

Anker, S.D., Swan, J.W., Volterrani, M., Chua, T.P., Clark, A.L., Poole-Wilson, P.A., Coats, A.J.S., 1997. The influence of muscle mass, strength, fatigability and blood flow on exercise capacity in cachectic and non-cachectic patients with chronic heart failure. Eur. Heart J. 18 (2), 259-269.

Arnold, E.M., Ward, S.R., Lieber, R.L., Delp, S.L., 2010. A model of the lower limb for analysis of human movement. Ann. Biomed. Eng. 38 (2), 269-279.

Barber, L., Barrett, R., Lichtwark, G., 2009. Validation of a freehand 3D ultrasound system for morphological measures of the medial gastrocnemius muscle. J. Biomech. 42 (9), 1313-1319.

Beneke, R., Meyer, K., 1997. Walking performance and economy in chronic heart failure patients pre and post exercise training. Eur. J. Appl. Physiol. 75 (3) 246-251.

Besier, T.F., Sturnieks, D.L., Alderson, J.A., Lloyd, D.G., 2003. Repeatability of gait data using a functional hip joint centre and a mean helical knee axis. J. Biomech. 36 (8), 1159-1168.
Carrington, C.A., Fisher, W.J., Davies, M.K., White, M.J., 2001. Is there a relationship between muscle fatigue resistance and cardiovascular responses to isometric exercise in mild chronic heart failure? Eur. J. HeartFailure 3 (1), 53-58.

Cicoira, M., Zanolla, L., Franceschini, L., Rossi, A., Golia, G., Zamboni, M., Tosoni, P., Zardini, P., 2001. Skeletal muscle mass independently predicts peak oxygen consumption and ventilatory response during exercise in noncachectic patients with chronic heart failure. J. Am. Coll. Cardiol. 37 (8), 2080-2085.

Clark, A.L., Poole-Wilson, P.A., Coats, A.J., 1996. Exercise limitation in chronic heart failure: central role of the periphery. J. Am. Coll. Cardiol. 28 (5), 1092-1102.

Cohn, J.N., Johnson, G.R., Shabetai, R., Loeb, H., Tristani, F., Rector, T., Smith, R., Fletcher, R., 1993. Ejection fraction, peak exercise oxygen consumption, cardiothoracic ratio, ventricular arrhythmias, and plasma norepinephrine as determinants of prognosis in heart failure. Circulation 87 (6 S), 15-16.

Delp, S.L., Arnold, A.S., Loan, P., Habib, A., John, C.T., Guendelman, E., Thelen, D.G., 2007. OpenSim: open-source software to create and analyze dynamic simulations of movement. IEEE Trans. Biomed. Eng. 54 (11), 1940-1950.

DeVita, P., Hortobagyi, T., 2000. Age causes a redistribution of joint torques and powers during gait. J. Appl. Physiol. 88 (5), 1804-1811.

Farris, D.J., Sawicki, G.S., 2012. The mechanics and energetics of human walking and running: A joint level perspective. J. R. Soc. Int. 9 (66), 110-118.

Figueiredo, P., Ribeiro, P.A., Bona, R.L., Peyré-Tartaruga, L.A., Ribeiro, J.P., 2013. Ventilatory determinants of self-selected walking speed in chronic heart failure. Med. Sci. Sports Exerc. 45 (3), 415-419.

Forman, D.E., Fleg, J.L., Kitzman, D.W., Brawner, C.A., Swank, A.M., McKelvie, R.S., Clare, R.M., Ellis, S.J., Dunlap, M.E., Bittner, V., 2012. 6-min walk test provides prognostic utility comparable to cardiopulmonary exercise testing in ambulatory outpatients with systolic heart failure. J. Am. Coll. Cardiol. 60 (25), 2653-2661.

Fülster, S., Tacke, M., Sandek, A., Ebner, N., Tschöpe, C., Doehner, W., Anker, S.D., von Haehling, S., 2013. Muscle wasting in patients with chronic heart failure: results from the studies investigating comorbidities aggravating heart failure (SICA-HF). Eur. Heart J. 34 (7), 512-519.

Harridge, S.D., Magnusson, G., Gordon, A., 1996. Skeletal muscle contractile characteristics and fatigue resistance in patients with chronic heart failure. Eur. Heart J. 17(6), 896-901.

Harrington, D., Anker, S.D., Chua, T.P., Webb-Peploe, K.M., Ponikowski, P.P., Poole-Wilson, P.A., Coats, A.J., 1997. Skeletal muscle function and its relation to exercise tolerance in chronic heart failure. J. Am. Coll. Cardiol. 30 (7), 1758-1764.

Juenger, J., Schellberg, D., Kraemer, S., Haunstetter, A., Zugck, C., Herzog, W., Haass, M., 2002. Health related quality of life in patients with congestive heart failure: comparison with other chronic diseases and relation to functional variables. Heart 87, 235-241.

Lipkin, D.P., Jones, D.A., Round, J.M., Poole-Wilson, P.A., 1988. Abnormalities of skeletal muscle in patients with chronic heart failure. Int. J. Cardiol. 18 (2), 187-195.

McGowan, C.P., Kram, R., Neptune, R.R., 2009. Modulation of leg muscle function in response to altered demand for body support and forward propulsion during walking. J. Biomech. 42 (7), 850-856.

Minotti, J.R., Pillay, P., Oka, R., Wells, L., Christoph, I., Massie, B.M., 1993. Skeletal muscle size: relationship to muscle function in heart failure. J. Appl. Physiol. 75 (1), 373-381.

Panizzolo, F.A., Green, D.J., Lloyd, D.G., Maiorana, A.J., Rubenson, J., 2013. Soleus fascicle length changes are conserved between young and old adults at their preferred walking speed. Gait \& Posture 38 (4), 764-769.

Panizzolo, F.A., Maiorana, A.J., Naylor, L.H., Lichtwark, G., Dembo, L., Lloyd, D.G., Green, D.J., Rubenson, J., 2014. Is the soleus a sentinel muscle for impaired aerobic capacity in heart failure? Med. Sci. Sports Exerc. (In press).

Riley, M., McParland, J., Stanford, C.F., Nicholls, D.P., 1992. Oxygen consumption during corridor walk testing in chronic cardiac failure. Eur. Heart J. 13 (6), 789-793.

Rubenson, J., Pires, J.N., Loi, H.O., Pinniger, G.J., Shannon, D.G., 2012. On the ascent: the soleus muscle is conserved to the ascending limb of the force length curve across gait mechanics in humans. J. Exp. Biol. 215 (20), 3539-3551.

Silder, A., Heiderscheit, B., Thelen, D.G., 2008. Active and passive contributions to joint kinetics during walking in older adults. J. Biomech. 41 (7), 1520-1527.

Sullivan, M.J., Green, H.J., Cobb, F.R., 1990. Skeletal muscle biochemistry and histology in ambulatory patients with long-term heart failure. Circulation 81 (2), 518-527.

Toth, M.J., Ades, P.A., Tischler, M.D., Tracy, R.P., LeWinter, M.M., 2006. Immune activation is associated with reduced skeletal muscle mass and physical function in chronic heart failure. Int. J. Cardiol. 109 (2), 179-187.

Toth, M.J., Shaw, A.O., Miller, M.S., VanBuren, P., LeWinter, M.M., Maughan, D.W., Ades, P.A., 2010. Reduced knee extensor function in heart failure is not explained by inactivity. Int. J. Cardiol. 143 (3), 276-282.

Treece, G.M., Gee, A.H., Prager, R.W., Cash, C.J.C., Berman, L., 2003. High definition freehand 3D ultrasound. Ultrasound Med. Biol. 29(4), 529-546. 